\title{
Fruit or fruit loops for fat loss; which would you choose?
}

\section{Opinion}

First of all, this is a trick question. Both Fruit \& Fruit Loops are comprised mainly of fructose in one form or another, either the pure form or in high fructose corn syrup.

Anyways, the answer might be surprising when you're looking to keep the weight off. You and I both know that they are both chock-full of sugar, but usually one decides to opt for the fruit. This is probably not the best choice, as the effects of all types of sugar have different repercussions on activating fatty acid synthesis. Trying to understand weight gain across a variety of body types and genetic predisposition to certain diseases is complex, lying beyond such obvious factors as glycemic index and coercing the biochemical community to take a closer look at metabolic pathways of specific macronutrients.

If you think of where your Fruit Loops go after they are digested, it will depend on the state of your skeletal muscle amongst other things. The glucose from your cereal can be converted to glycogen and stored in your muscle bellies for functional energy, especially if you are an avid gym-goer or work out often. Fructose, on the other hand, has to be converted into glucose and then into glycogen, of which we do not know the percentage of this double conversion that actually takes place. One of the major differentiators between glucose and fructose metabolism is the location of its breakdown; glucose happens all over the body where fructose is solely in the liver. Let's understand more.

One study in particular, Comparative Effects of Fructose and Glucose on Lipogenic Gene Expression and Intermediary Metabolism in HepG2 Liver Cells, ${ }^{1}$ makes a valid assumption that all wellness practitioners should consider-high fructose consumption is directly correlated to fatty-acid synthesis, more so than glucose, however not from the mechanism of gene expression.

Following fructose consumption, there are high amounts of both carbon, pyruvate \& triose-phosphates, both major components of de novo lipogenesis along with the fact that fructose metabolism bypasses the necessity of phosphofructokinase, an enzyme needed for glycolysis. That seems to be the cause of the high amount of lipogenesis taking place in the presence of fructose moreso than other sugars.

Another study by Patrick Lam out of Dr. Marc Hellerstein's research lab at Berkeley, Effects of Consuming Dietary Fructose versus Glucose on de novo Lipogenesis in Overweight and Obese Human Subjects, ${ }^{2}$ confirms this assumption. After a 10 week study on 10 subjects, fed either glucose or fructose sweetened drinks
Volume 3 Issue 3 - 2016

\author{
Kim Klafczynski \\ Renew U, USA
}

Correspondence: Kim Klafczynski, Renew U Founder \& Wellness Advocate, Renew U, USA, Email kim@renewufit.com

Received: January 08, 2016 | Published: January II, 2016

comprising of $25 \%$ of their daily caloric intake, de novo lipogenesis increased $6 \%$ of the fructose-ingesting group over those drinking the glucose. In a fed state, those ingesting fructose-containing drinks for a sustained period had significantly higher de novo lipogenesis.

As an article for thought, keep an open mind as you reach for that daily lunch apple or banana with your breakfast; as high fructose corn syrup overtakes many store brands, the same effect is had with fruit in general. For a safe alternative to continue keeping the extra weight at bay, opt for green veggies and hummus or a salad with romaine \& sunflower seeds. As more research surfaces on the topic of fructolysis, we will all become more informed on the repercussion of fruit on fighting the obesity epidemic $\&$ the drivers that are causing fructose lipogenesis to be so high.

\section{Acknowledgements}

None.

\section{Conflict of interest}

Author declares that there is no conflict of interest.

\section{References}

1. Hirahatake KM, Meissen JK, Fiehn O, et al. Comparative Effects of Fructose and Glucose on Lipogenic Gene Expression and Intermediary Metabolism in HepG2 Liver Cells. PloS One. 2011;6(11):e26583.

2. Patrick L. Effects of Consuming Dietary Fructose versus Glucose on De Novo Lipogenesis in Overweight and Obese Human Subjects. Berkeley Scientific Journal. 2011;15(2). 